\title{
CONTROLE DE VELOCIDADE SEM SENSOR MECÂNICO DE UMA MÁQUINA ASSÍNCRONA UTILIZANDO A ESTRATÉGIA DE CONTROLE DTC
}

\author{
Fabiano Salvadori $^{\dagger}$, Gideon V. Leandro ${ }^{\dagger}$, Antonio M.N. Lima ${ }^{\ddagger}$ e Cursino B. Jacobina ${ }^{\ddagger}$ \\ $\dagger$ Universidade Regional do Noroeste do Estado do RGS - DeTEC/EGE \\ \{fabiano,gede\}@unijui.tche.br \\ Caixa Postal 560, 98.700-000 Ijuí, RS, Brasil \\ ‡ Universidade Federal de Campina Grande - UFCG/DEE \\ \{amnlima,jacobina\}@dee.ufcg.edu.br \\ Caixa Postal 10.105, 58109-970 Campina Grande, PB, Brasil
}

\begin{abstract}
Resumo - O controle direto de conjugado (Direct Torque Control - DTC) é uma alternativa para o controle de fluxo e conjugado de máquinas elétricas, pois alia robustez com simplicidade de implementação. Esta estratégia utiliza o vetor fluxo estatórico para controlar a magnetização da máquina e o escorregamento para controlar o conjugado eletromagnético. Além disso a implementação do DTC é relativamente independente dos parâmetros rotóricos da máquina, funciona sem sensor mecânico de velocidade ou posição e permite controlar a tensão aplicada na máquina diretamente sem a necessidade de uma malha intermediários de controle de corrente. Neste trabalho é proposto o controle de velocidade, sem sensor mecânico, em acionamentos com máquinas assíncronas utilizando a estratégia $D T C$. A velocidade é estimada utilizandose a freqüência angular de escorregamento e a freqüência angular síncrona do vetor fluxo estatórico na saída da malha de controle de conjugado. Resultados de simulações e experimentais são apresentados e discutidos.
\end{abstract}

Abstract - The Direct Torque Control (DTC) is an alternative for flux and torque control of electrical machines, since it combines robustness and simplicity of implementation. This strategy uses the stator flux vector to control the machine magnetization and the slip frequency to control the eletromagnetic torque. Moreover, the DTC implementation is relatively independent from the rotor parameters, and can be used without a mechanical speed or position sensor and allows to directly control the voltage supplied to the machine without the need of an intermediate current control loop. The speed sensorless control for asynchronous machines by using the DTC strategy is proposed in this paper. The rotor speed is estimated by the use of the angular slip frequency and the angular synchronous frequency of the stator flux vector. Results of the simulations and experiments are shown and discussed.

\section{INTRODUÇÃO}

Sistemas de acionamentos elétricos estáticos utilizando máquinas assíncronas são empregados industrialmente em controles de movimento de sistemas mecânicos há muito tempo, isto porque, são mecanicamente robustos. Contudo sua análise é complexa pois requer o estudo de um sistema multivariável e não linear. Inicialmente, eram utilizados apenas em aplicações de grande porte e onde não era necessário um controle muito preciso, seja de conjugado, velocidade ou posição.

Os primeiros esquemas de acionamentos com máquina assíncrona eram do tipo escalar e baseados em modelos de regime permanente, tal como o Volts/Hertz [1], apresentando um fraco desempenho dinâmico. No intuito de desenvolver sistemas de acionamento de alto desempenho, têm sido investigadas estratégias de controle que assegurem o desacoplamento entre as variáveis finais a serem controladas, no caso o fluxo e o conjugado. A utilização de técnicas genéricas de desacoplamento de sistemas, tal como proposto por Falb e Wollovich [2], ou baseados em modelos escalares, como proposto por Bose [3], levam em geral a soluções pouco eficazes e eventualmente complexas. Entretanto, explorando convenientemente o modelo da máquina, é possível obter este desacoplamento utilizando abordagens ditas vetoriais [4], [5] e [6].

As técnicas de controle das máquinas assíncronas propostas na literatura podem ser classificadas basicamente em duas categorias: controle escalar e controle vetorial. No controle vetorial citam-se: Controle com Orientação Indireta pelo Campo (Indirect Field Oriented Control - IFOC); Controle com Orientação Direta pelo Campo (Direct Field Oriented Control - DFOC); e Controle Direto de Conjugado (Direct Torque Control - DTC).

O Controle $D T C$, na forma como é conhecido, foi proposto inicialmente por Takahashi e Noguchi [7], denominado $D i$ rect Torque Control - DTC, e apresentado por Depenbrock [8] como Direct Self Control - DSC, direcionado para acionamentos com máquina assíncronas alimentados por inversores fonte de tensão aplicado principalmente em sistemas de tração mecânica onde o controle do conjugado é a grandeza principal a ser controlada.

$\mathrm{O}$ método foi então generalizado, por Boldea e Nasar [9] para acionamentos com máquina assíncronas com alimentação em corrente, acionamentos com máquinas síncronas alimentadas em tensão e corrente; por Habetler e Divan [10] em estratégias DTC utilizando modulação por largura de pulso vetorial; e, por Mir et alii, [11] para estratégias utilizando controladores com lógica Fuzzy. Para efeitos de generalização passar-se-á a definir as estratégias de Controle Direto de Fluxo e Conjugado, como estratégias $D T C$. Usualmente, as estratégias DTC são empregadas em 
acionamentos onde deseja-se o controle de conjugado.

Neste trabalho é proposta uma estratégia $D T C$ com implementação discreta, utilizando controladores PI (Proporcional + Integral) e modulação PWM (Pulse Width Modulation) regular escalar simétrica aplicada ao controle de velocidade da máquina assíncrona sem sensor mecânico. A velocidade estimada é obtida subtraindo-se do valor da freqüência angular síncrona de referência, obtido na saída do controlador PI, o valor da freqüência de escorregamento estimada, calculado através do modelo dinâmico em regime permanente da máquina. A estratégia DTC já é utilizada em sistemas de acionamento comercializados pela ABB. A novidade introduzida neste trabalho é, de fato, sua associação com as técnicas de estimação propostas neste trabalho.

\section{MODELO DA MÁQUINA}

O modelo dinâmico elétrico, contínuo no tempo, da máquina assíncrona, na forma de equações diferenciais em coordenadas $d q$ num referencial genérico (sobrescrito $g$ ) é,

$$
\begin{aligned}
\mathbf{v}_{\mathbf{s}}^{\mathbf{g}} & =r_{s} \mathbf{i}_{\mathbf{s}}^{\mathbf{g}}+\frac{d}{d t} \phi_{\mathbf{s}}^{\mathbf{g}}+j \omega_{g} \phi_{\mathbf{s}}^{\mathbf{g}} \\
0 & =r_{r} \mathbf{i}_{\mathbf{r}}^{\mathbf{g}}+\frac{d}{d t} \phi_{\mathbf{r}}^{\mathbf{g}}+j\left(\omega_{g}-\omega_{r}\right) \phi_{\mathbf{r}}^{\mathbf{g}}
\end{aligned}
$$

e as respectivas expressões representativas do modelo mecânico

$$
\begin{aligned}
P\left(C_{e}-C_{c}\right) & =J_{m} \frac{d}{d t} \omega_{r}+F_{a} \omega_{r} \\
C_{e} & =P l_{m}\left(\mathfrak{I m}\left(i_{s}^{g}\left(i_{r}^{g}\right)^{\#}\right)\right) \\
\frac{d}{d t} \delta_{r} & =\omega_{r}
\end{aligned}
$$

Efetivando-se as transformações adequadas, obtém-se as expressões dos fluxos e correntes estatóricas e rotóricas no referencial genérico,

$$
\begin{aligned}
\phi_{\mathbf{s}}^{\mathrm{g}} & =l_{s} \mathbf{i}_{\mathbf{s}}^{\mathbf{g}}+l_{m} \mathbf{i}_{\mathbf{r}}^{\mathbf{g}} \\
\phi_{\mathbf{r}}^{\mathbf{g}} & =l_{r} \mathbf{i}_{\mathbf{r}}^{\mathbf{g}}+l_{m} \mathbf{i}_{\mathbf{s}}^{\mathbf{g}} \\
\mathbf{i}_{\mathbf{s}}^{\mathbf{g}} & =\frac{\phi_{\mathbf{s}}^{\mathbf{g}}}{\sigma l_{s}}-\frac{l_{m} \phi_{\mathbf{r}}^{\mathbf{g}}}{\sigma l_{s} l_{r}} \\
\mathbf{i}_{\mathbf{r}}^{\mathbf{g}} & =\frac{\phi_{\mathbf{r}}^{\mathbf{g}}}{\sigma l_{r}}-\frac{l_{m} \phi_{\mathbf{s}}^{\mathbf{g}}}{\sigma l_{s} l_{r}}
\end{aligned}
$$

onde $\sigma=1-\frac{l_{m}^{2}}{l_{s} l_{r}}$ é o coeficiente de dispersão.

Neste conjunto de equações, o índice $g$ é usado para indicar que as equações encontram-se num sistema genérico de coordenadas.

$\mathrm{Na}$ figura 1 são apresentados os vetores instantâneos para as variáveis $\mathbf{v}_{s}^{s}, \mathbf{i}_{s}^{s}, \phi_{s}^{s}$, e $\phi_{r}^{s}$ vistos do referencial estatórico (fase $s_{1}$ ). Também nessa figura são indicados o eixo magnético rotórico (fase $r_{1}$ ) e o eixo $d$.

O diagrama vetorial instantâneo da máquina mostrando as relações relevantes para compreensão da estratégia de controle é mostrado na figura 1.

As grandezas são representadas na forma: $\mathbf{v}_{\mathbf{s}}^{\mathbf{g}}=v_{s d}^{g}+j v_{s q}^{g}$ - vetor tensão estatórica; $\mathbf{i}_{\mathbf{s}}^{\mathbf{g}}=i_{s d}^{g}+j i_{s q}^{g}$ - vetor corrente estatórica; $\mathbf{i}_{\mathbf{s} 123}$ - vetor corrente estatórica trifásica; $\phi_{\mathrm{s}}^{\mathrm{g}}=$ $\phi_{s d}^{g}+j \phi_{s q}^{g}$ - vetor fluxo estatórico; $\phi_{s}, i_{s}$ - magnitude fluxo e corrente estatórica; $\omega_{a}, \delta_{a}$ - freqüência angular/ângulo vetor fluxo estatórico; $\omega_{r}, \delta_{r}$ - velocidade rotórica/ângulo

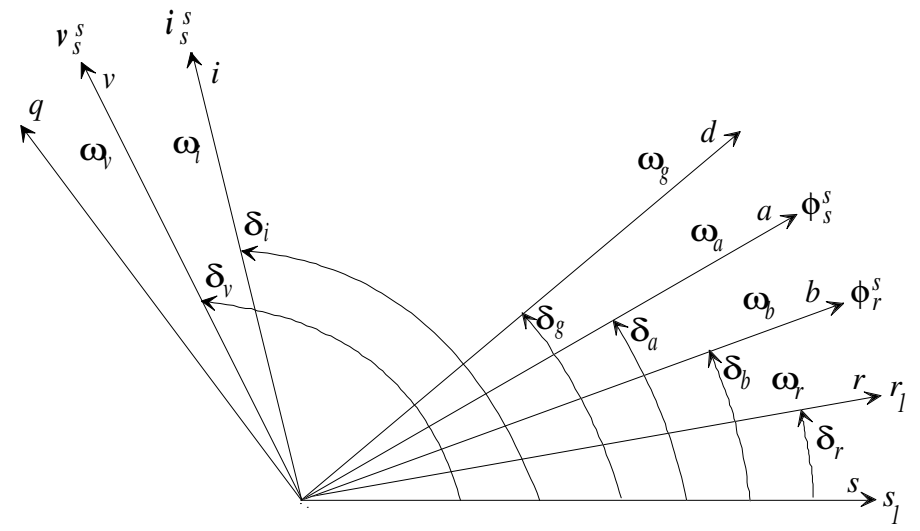

Figura 1 Diagrama vetorial instantâneo da máquina.

eixo rotórico; $\omega_{g}, \delta_{g}$ - freqüência angular/ângulo eixos $d q$; $\omega_{b}, \delta_{b}$ - freqüência angular/ângulo vetor fluxo rotórico; $\omega_{i}, \delta_{i}$ - freqüência angular/ângulo vetor corrente estatórica; $\omega_{v}, \delta_{v}$ - freqüência angular/ângulo vetor tensão estatórica; $C_{e}, C_{l}$ conjugados eletromagnético e de carga; $P, J_{m}$ e $F_{a}$ - número de pares de pólos, constante de inércia e coeficiente de atrito; $r_{s}$ e $r_{r}$ - resistências estatórica e rotórica; $l_{s}, l_{r}$ e $l_{m}$ - indutâncias estatórica, rotórica e mútua; $\mathfrak{I m}$ - parcela imaginária do vetor; *, ^ e \#-grandezas de referência, estimadas e conjugado complexo. Definição vetorial similar é aplicada para as variáveis rotóricas substituindo o subscrito $s$ pelo subscrito $r$.

\section{ESTIMAÇÃO DE FLUXO E CONJUGADO}

Para as estratégias de controle DTC, é importante o processo de estimação de estados pois as malhas de controle são o vetor fluxo estatórico e o conjugado eletromagnético, obtidos através de estimadores, cujas grandezas de entrada são corrente e tensão. Além disso, as malhas de controle de fluxo e conjugado, são os blocos internos para o controle de velocidade, um bom controle de fluxo e conjugado é fator fundamental para um correto controle de velocidade.

A estimação de estados é baseada no modelo matemático disponível da máquina, um conjunto de seis equações diferenciais não lineares, acrescida do fato que a resistência rotórica da máquina varia com o tempo de funcionamento, devido ao aquecimento da máquina.

A maneira mais comum de obtenção do vetor fluxo estatórico é através da expressão da tensão estatórica (modelo em tensão) (Salvadori et alii, [12]). No eixo de referência estatórico $\left(\omega_{g}=0, g=s\right)$ pode-se obter os vetores do fluxo estatórico e fluxo rotórico estimado pelo cálculo das expressões

$$
\begin{aligned}
& \widehat{\phi}_{\mathbf{s}}^{\mathbf{s}}=\int_{0}^{t}\left(\mathbf{v}_{\mathbf{s}}^{\mathbf{s}}(\tau)-r_{s} \mathbf{i}_{\mathbf{s}}^{\mathbf{s}}(\tau)\right) d \tau \\
& \widehat{\phi}_{\mathbf{r}}^{\mathbf{s}}=\frac{l_{r}}{l_{m}}\left(\widehat{\phi}_{\mathbf{s}}^{\mathbf{s}}-\sigma l_{s} \mathbf{i}_{\mathbf{s}}^{\mathbf{s}}\right)
\end{aligned}
$$

Esta abordagem independe da posição rotórica ou informação da velocidade. Contudo, apresenta problemas de integração e, na operação em velocidade próxima de zero a queda de tensão na resistência estatórica torna-se significativa, devido ao fato que os integradores não operam bem em velocidade zero pois não existe FEM (Força Eletromotriz) 
induzida na máquina. A estimação imprecisa do fluxo estatórico, devido a variação da resistência estatórica, pode tornar o controle instável. Portanto, a utilização de um método mais sofisticado para a estimação de estados pode ser interessante, ou então, um método que corrija as variações da resistência estatórica. Em altas velocidades o modelo em tensão é menos sensível a variações paramétricas porque a FCEM (Força Contra-Eletromotriz) tende a dominar.

Como forma de contornar estes problemas e acelerar a convergência do estimador de estados é possível a implementação de um observador de ordem completa, que consiste basicamente na simulação em tempo real com correção através da realimentação de erro. Contudo, a implementação em tempo real de um observador de ordem completa é problemática em virtude do tempo necessário para discretização do modelo da máquina.

Portanto, o observador implementado foi o modelo em tensão e o conjugado eletromagnético pode ser estimado através da expressão,

$$
\widehat{C}_{e}=\frac{P l_{m}}{\sigma l_{s} l_{r}}\left(\widehat{\phi}_{s q}^{s} \widehat{\phi}_{r d}^{s}-\widehat{\phi}_{s d}^{s} \widehat{\phi}_{r q}^{s}\right)
$$

ou então através de $([13])$

$$
\widehat{C}_{e} \cong \frac{P \omega_{a r} l_{m}^{2}\left(\widehat{\phi}_{s}^{s}\right)^{2}}{r_{r} l_{s}^{2}}
$$

Lembrando que a expressão (13) é aproximada.

\section{O CONTROLE DTC-FEE}

A estratégia de controle DTC a Fluxo Estatórico por Escorregamento no Estator (DTC-FEE), pode ser visualizada na figura 2. Apresentada inicialmente por Salvadori et. alii, [12] a estratégia consiste em controlar a magnetização da máquina através do fluxo estatórico, normalmente num valor constante (exceto nos casos de enfraquecimento de campo e otimização da eficiência da máquina) e o conjugado eletromagnético através da freqüência de escorregamento da variável escolhida para excitar a máquina (neste caso o fluxo estatórico). Sua implementação é simples, totalmente discreta, utilizando controladores PI convencionais. Apresenta resposta dinâmica rápida a degraus de fluxo e conjugado com reduzido ripple em regime permanente, baixa sensibilidade paramétrica e robustez de funcionamento.

Considerando que o referencial para o modelo de atuação é o estatórico, onde $\omega_{g}=0$ e $\delta_{g}=0$, a matriz de transformação das grandezas trifásicas para bifásicas resulta,

$$
T\left(\delta_{g}\right)=\sqrt{\frac{2}{3}}\left[\begin{array}{ccc}
\cos (0) & \cos \left(-\frac{2 \pi}{3}\right) & \cos \left(\frac{2 \pi}{3}\right) \\
-\operatorname{sen}(0) & -\operatorname{sen}\left(-\frac{2 \pi}{3}\right) & -\operatorname{sen}\left(\frac{2 \pi}{3}\right)
\end{array}\right]
$$

A estratégia incorpora um controlador PI na malha externa de controle do conjugado. O conjugado estimado comparado com o conjugado de referência (saída do controlador PI da malha de velocidade), determina o erro de conjugado cujo sinal através de um controlador PI, gera a freqüência angular síncrona de referência que deverá ser aplicada à máquina de maneira a obter a velocidade desejada. A referência do conjugado eletromagnético pode ser definida diretamente ou através da implementação de uma malha de controle de velocidade como descrito acima. O controle de fluxo e conjugado é feito diretamente das referências das variáveis de controle $\phi_{s}^{*}$ e $C_{e}^{*}$ para tensão estatórica, sem estágio intermediário para controle de corrente (fonte de corrente).

O ponto chave do controle reside no fato de manter-se o campo magnético estatórico constante e que a freqüência angular de rotação de referência $\omega_{a}^{*}$ ajuste-se de forma que um valor de conjugado desejado seja produzido. Quando a magnetização da máquina é mantida constante através do controle do fluxo estatórico, todas as outras variáveis (tensões, correntes e conjugado) são funções somente da freqüência de escorregamento. Escolhendo uma freqüência de escorregamento que acelere ou desacelere a rotação do vetor fluxo estatórico, possibilita-se controlar o conjugado como desejado.

A partir da função de transferência em malha fechada é possível determinar as características desejadas para as respostas do sistema (fluxo e conjugado) em condições de regime transitório e permanente.

Para o projeto dos controladores, blocos 1, 2, e 5, da figura 2, dentre os vários modelos disponíveis, foram utilizados dois modelos dinâmicos descritos a seguir. O primeiro deles consiste em aproveitar a equação de tensão estatórica (1) diretamente com o termo da queda de tensão resistiva tomado como perturbação a ser compensada.

$$
\mathbf{v}_{\mathbf{s}}^{\mathbf{g}}=r_{s} \mathbf{i}_{\mathbf{s}}^{\mathbf{g}}+\frac{d}{d t} \phi_{\mathbf{s}}^{\mathbf{g}}+j \omega_{g} \phi_{\mathbf{s}}^{\mathbf{g}}
$$

decompondo em componentes $d, q$

$$
\begin{aligned}
& v_{s d}^{g}=r_{s} i_{s d}^{g}+\frac{d}{d t} \phi_{s d}^{g}-\omega_{g} \phi_{s q}^{g} \\
& v_{s q}^{g}=r_{s} i_{s q}^{g}+\frac{d}{d t} \phi_{s q}^{g}+\omega_{g} \phi_{s d}^{g}
\end{aligned}
$$

O segundo modelo, é obtido pela substituição do vetor corrente estatórica (8) na expressão (1) resultando na expressão da tensão estatórica função dos fluxos estatórico e rotórico, definindo o seguinte modelo dinâmico,

$$
\mathbf{v}_{\mathbf{s}}^{\mathbf{g}}=\frac{1}{\sigma \tau_{s}} \phi_{\mathbf{s}}^{\mathbf{g}}+\frac{d \phi_{\mathbf{s}}^{\mathbf{g}}}{d t}+j \omega_{g} \phi_{\mathbf{s}}^{\mathbf{g}}-\frac{l_{m}}{\sigma \tau_{s} l_{r}} \phi_{\mathbf{r}}^{\mathbf{g}}
$$

onde $\tau_{s}=\frac{l_{s}}{r_{s}}$ é a constante de tempo estatórica. Decompondo em componentes $d, q$

$$
\begin{aligned}
v_{s d}^{g} & =\frac{1}{\sigma \tau_{s}} \phi_{s d}^{g}+\frac{d}{d t} \phi_{s d}^{g}-\omega_{g} \phi_{s q}^{g}-\frac{l_{m}}{\sigma \tau_{s} l_{r}} \phi_{r d}^{g} \\
v_{s q}^{g} & =\frac{1}{\sigma \tau_{s}} \phi_{s q}^{g}+\frac{d}{d t} \phi_{s q}^{g}+\omega_{g} \phi_{s d}^{g}-\frac{l_{m}}{\sigma \tau_{s} l_{r}} \phi_{r q}^{g}
\end{aligned}
$$

Existem preferencialmente duas alternativas para implementação dos controladores do fluxo estatórico. A primeira delas, consiste em utilizar o estator fixo $\left(\omega_{g}=0\right)$ como referencial para o modelo de atuação; e a segunda alternativa, utilizar o referencial síncrono $\left(\omega_{g}=\omega_{a}\right)$.

Neste trabalho, optou-se pelo modelo da equação (18) no referencial fixo para evitar os termos de acoplamento entre as componentes de fluxo estatórico eixos $d, q$, apesar de que, com esta opção, as variáveis a controlar serem alternadas. Com isto a expressão (18) resulta,

$$
\mathbf{v}_{\mathbf{s}}^{\mathbf{s}}=\frac{1}{\sigma \tau_{s}} \phi_{\mathbf{s}}^{\mathbf{s}}+\frac{d \phi_{\mathbf{s}}^{\mathbf{s}}}{d t}-\frac{l_{m}}{\sigma \tau_{s} l_{r}} \phi_{\mathbf{r}}^{\mathbf{s}}
$$


Neste caso, a partir de (21), tem-se as seguintes equações dinâmicas, em coordenadas $d, q$, referencial fixo,

$$
\begin{aligned}
v_{s d}^{s} & =\frac{1}{\sigma \tau_{s}} \phi_{s d}^{s}+\frac{d}{d t} \phi_{s d}^{s}-\frac{l_{m}}{\sigma \tau_{s} l_{r}} \phi_{r d}^{s} \\
v_{s q}^{s} & =\frac{1}{\sigma \tau_{s}} \phi_{s q}^{s}+\frac{d}{d t} \phi_{s q}^{s}-\frac{l_{m}}{\sigma \tau_{s} l_{r}} \phi_{r q}^{s}
\end{aligned}
$$

e os termos a compensar, bloco 3 , figura 2, são perturbações (forças contra-eletromotrizes rotóricas),

$$
\begin{aligned}
e_{d}^{s} & =-\frac{l_{m}}{\sigma \tau_{s} l_{r}} \phi_{r d}^{s} \\
e_{q}^{s} & =-\frac{l_{m}}{\sigma \tau_{s} l_{r}} \phi_{r q}^{s}
\end{aligned}
$$

Para os controles a fluxo estatórico constante, o desacoplamento entre os comandos de fluxo e conjugado somente é válido abaixo do valor de pull-out de conjugado, região onde é possível controlar $\phi_{s}$ e $C_{e}$ independentemente [14] o que impossibilita um perfeito desacoplamento. O escorregamento é representado por

$$
\omega_{a r}^{*}=\left(\frac{1-\sigma}{2 \sigma^{2} \tau_{r} l_{s}}\right) \frac{P\left(\phi_{s}^{*}\right)^{2}}{C_{e}} \pm \sqrt{\left(\frac{1-\sigma}{2 \sigma^{2} \tau_{r} l_{s}}\right)^{2}-\frac{1}{\sigma^{2} \tau_{r}}}
$$

O termo em (26) é a solução da equação de segundo grau cujo valor particular (sinal \pm ) corresponde a um ponto de operação possível da máquina assíncrona. O conjugado de pull-out, para um dado fluxo estatórico, corresponde ao valor máximo de operação de $\omega_{a r}$, obtido quando o radicando é igual a zero.

Quando a excitação magnética é controlada pelo fluxo estatórico, a expressão de conjugado eletromagnético, obtida de (4), (6)-(9), é,

$$
C_{e} \cong \frac{P \omega_{a r} l_{m}^{2} \phi_{s}^{2}}{r_{r} l_{s}^{2}\left(1+\omega_{a r} \tau_{r}\right)}
$$

onde $\omega_{a r}\left(=\omega_{a}-\omega_{r}\right)$ é a freqüência de escorregamento. Considerando $\omega_{a r} \tau_{r}<<1$ a expressão de conjugado em regime permanente abaixo do valor de "pull-out" resulta na forma aproximada apresentada em (13).

O erro de conjugado gerado passa pelo controlador PI cuja saída é a "imagem" da freqüência angular de referência $\omega_{a}^{*}$, desejada ou imposta, para aceleração/desaceleração do vetor fluxo estatórico. O controlador procura "compensar" o erro de conjugado aumentando/diminuindo o valor de $\omega_{a}^{*}$ consequentemente acelerando/desacelerando o vetor fluxo. O erro de conjugado é proporcional a $\omega_{a}^{*}$ portanto, alterar a freqüência angular síncrona significa alterar o escorregamento e vice-versa. Integrando $\omega_{a}^{*}$ obtém-se o ângulo de transformação (ou desacoplamento),

$$
\delta_{a}^{*}=\int_{0}^{t} \omega_{a}^{*}(\tau) d \tau+\delta_{a}(0)
$$

é possível então a decomposição (desacoplamento) do vetor fluxo estatórico de referência em componentes $d, q$ no referencial fixo (bloco 4, figura 2).

$$
\begin{aligned}
\phi_{s}^{s *} & =\phi_{s}^{*} e^{j \delta_{a}^{*}} \\
\phi_{s d}^{s *} & =\phi_{s}^{*} \cos \left(\delta_{a}^{*}\right) \\
\phi_{s q}^{s *} & =\phi_{s}^{*} \operatorname{sen}\left(\delta_{a}^{*}\right)
\end{aligned}
$$

\section{O CONTROLE DTC DE VELOCIDADE SEM SENSOR MECÂNICO}

O conhecimento da velocidade é essencial no controle IFOC e pode ser necessário nos controles DFOC e DTC, quando é imprescindível o conhecimento dos fluxos seja através de medição ou através de estimação. No caso de estimação, dependendo do tipo de observador implementado, o conhecimento da velocidade é essencial (Jansen, [15]) e (Lorenz, [16]).

Usualmente utiliza-se sensores mecânicos acoplados ao eixo da máquina para a medição da posição ou velocidade. A utilização destes sensores apresenta uma série de desvantagens tais como, aumento do custo, diminuição da confiabilidade (baixa robustez mecânica) e baixa imunidade a ruídos deteriorando, de forma geral, o sistema de acionamento. Isto se contrapõe a uma das vantagens na utilização de máquinas assíncronas em acionamentos de alto desempenho que é sua robustez mecânica.

Com o desenvolvimento da microeletrônica e a contínua redução dos custos computacionais, a substituição dos sensores mecânicos por soluções baseadas em software (estimação) ganham impulso e tornam-se atrativas. Esta substituição objetiva tornar o sistema mais robusto acrescido do fato de torná-lo, economicamente, menos oneroso.

Ribeiro et alii, [17], propõem uma classificação dos métodos de estimação de velocidade divididos em: (I) métodos baseados no modelo $d q$ por meio da força contraeletromotriz (FCEM) ou das equações de tensão estatórica da máquina e, (II) métodos baseados na medição dos harmônicos de corrente ou tensão produzidos por saliências da máquina.

I Métodos baseados no modelo $d q$ por meio da força contra-eletromotriz $(F C E M)$ ou das equações de tensão estatórica da máquina.

- estimação do escorregamento: regime permanente ou dinâmico;

- estimação da velocidade através do modelo de estado ou função de transferência;

- controle adaptativo tipo modelo de referência;

- filtro de Kalman;

II Métodos baseados na medição dos harmônicos de corrente ou tensão produzidos por saliências na máquina.

- baseados na alimentação fundamental;

- baseados na injeção de sinais de alta freqüência.

A figura 2 apresenta o diagrama de blocos esquemático da estratégia de controle proposta. A freqüência angular síncrona de referência, é obtida diretamente na saída do controlador PI, bloco 2, da figura 2. O escorregamento estimado é obtido, considerando o modelo $d q$ da máquina, de acordo com a expressão (32),

$$
\widehat{\omega}_{a r}=\frac{l_{s}^{2} r_{r} \widehat{C}_{e}}{P \widehat{\phi}_{s}^{2} l_{m}^{2}}=\frac{l_{s}^{2}\left(\frac{l_{r}}{\tau_{r}}\right) \widehat{C}_{e}}{P \widehat{\phi}_{s}^{2} l_{m}^{2}}
$$

A estimação da velocidade é realizada utilizando a relação entre a freqüência angular síncrona de referência do vetor fluxo estatórico $\omega_{a}^{*}$ e o escorregamento estimado (32), de 
acordo com a expressão,

$$
\widehat{\omega}_{r o}=\omega_{a}^{*}-\widehat{\omega}_{a r}
$$

o sinal de $\widehat{\omega}_{\text {ro }}$ passa então, por um processo de filtragem para eliminar o ripple existente utilizando um filtro discreto Butterworth de segunda ordem, resultado na freqüência rotórica estimada filtrada $\widehat{\omega}_{r}$

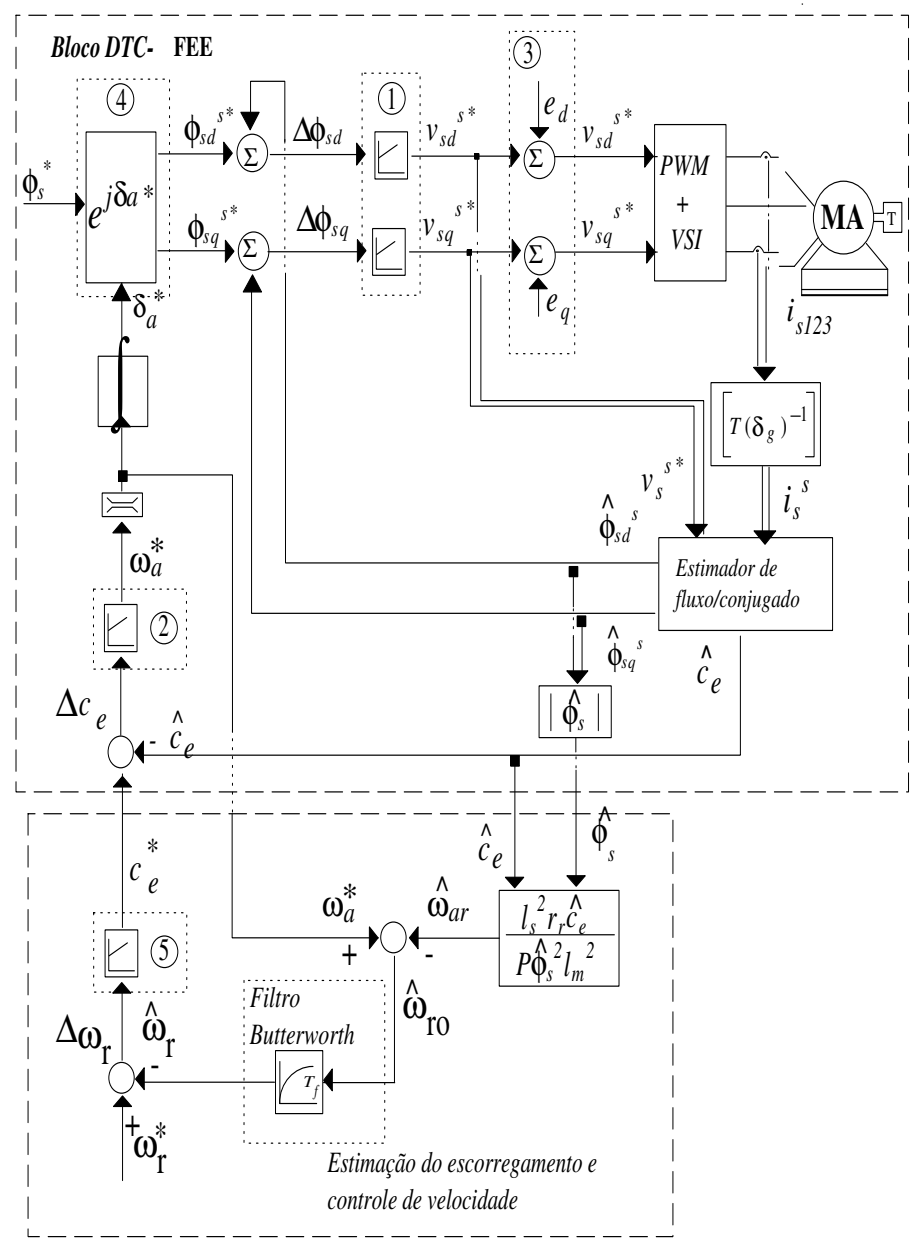

Figura 2 Diagrama esquemático DTC-FEE sem sensor mecânico de velocidade.

Este método de estimação da velocidade é dependente do conhecimento da magnitude do vetor fluxo estatórico estimado, do conjugado eletromagnético estimado; e dos parâmetros $l_{s}, \sigma l_{s}$ e $\tau_{r}$ parâmetros estes que podem ser estimados. A variação dos parâmetros envolvidos na estimação, tanto do fluxo estatórico como do conjugado, é compensada parcialmente pelos controladores PI de fluxo, bloco 1 da figura 2.

Comparativamente ao diagrama de blocos da estratégia $D T C$-FEE, observa-se que foram introduzidos:

1. Um bloco para o cálculo do escorregamento estimado;

2. Comparador das velocidades de referência e estimada;

3. Controlador PI de velocidade, projetado utilizando os mesmos critérios utilizados para o projeto dos controladores de fluxo;

4. Filtro Butterworth discreto de segunda ordem, com freqüência de corte de $1 \mathrm{KHz}$ para filtragem da velocidade estimada $\widehat{\omega}_{r}$.

\section{PROJETO DOS CONTROLADORES}

O modelo dinâmico de fluxo utilizado para o projeto dos controladores da estratégia apresentada são do tipo linear invariante no tempo. Este modelo é de primeira ordem onde assume-se que os termos de perturbação (FCEM) são constantes durante o intervalo de amostragem $\left(t_{a}\right)$. O período de amostragem é definido em função da máxima freqüência de chaveamento do inversor e levando em conta as constantes do sistema (máquina). O período de amostragem escolhido para as malhas de fluxo foi de $100 \mu \mathrm{s}$ e a da malha de velocidade 40 vezes maior, referente às tarefas de aquisição de dados, estimação de fluxo e conjugado e execução do algorítimo de controle.

Os controladores discretos utilizados são do tipo PI, calculados de modo a obter-se em malha fechada uma função de transferência de segunda ordem com coeficiente de amortecimento ótimo (Buhler, [18]). A função de transferência dos controladores é,

$$
G_{C}(s)=\frac{1+s T_{n}}{s T_{i}}
$$

onde: $T_{i}$ é a constante de integração e $T_{n} / T_{i}$ é o ganho proporcional.

Estes controladores são projetados de forma síncrona com o comando da fonte de tensão PWM. A figura 3, mostra o diagrama de blocos típico dos controladores projetados, neste caso, o de fluxo. Nesta figura, o bloco delimitado por linhas pontilhadas, corresponde a função de transferência de primeira ordem do sistema. Neste bloco, a função de transferência $G_{s}(s)$ corresponde a expressão (38), obtida de (35)-(37) e os termos $e_{d M}^{s}$ e $e_{q M}^{s}$ correspondem as expressões (24) e (25). Os blocos $R_{\varphi d}$ e $R_{\varphi q}$, correspondem aos controladores PI de fluxo estatórico, eixos $d, q$.

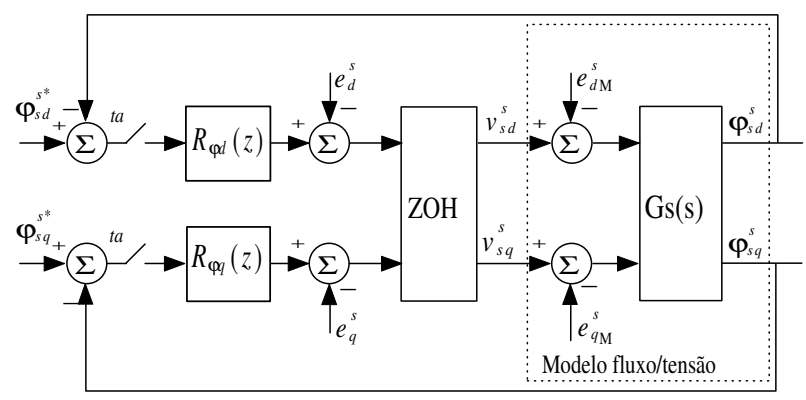

Figura 3 Sistema de controle típico.

Aplicando a transformada de Laplace em (21) tem-se

$$
\begin{aligned}
\left(V_{s}^{s}(s)+\frac{l_{m}}{\sigma \tau_{s} l_{r}} \boldsymbol{\Phi}_{\mathbf{r}}^{\mathbf{s}}\right) & =\frac{1}{\sigma \tau_{s}} \boldsymbol{\Phi}_{\mathbf{s}}^{\mathbf{s}}(s)+s \boldsymbol{\Phi}_{\mathbf{s}}^{\mathbf{s}}(s) \\
V_{s}^{s}(s)^{\prime} & =V_{s}^{s}(s)+\frac{l_{m}}{\sigma \tau_{s} l_{r}} \boldsymbol{\Phi}_{\mathbf{r}}^{\mathbf{s}} \\
G_{S}(s) & =\frac{\boldsymbol{\Phi}_{\mathbf{s}}^{\mathbf{s}}(s)}{V_{s}^{s}(s)^{\prime}}=\frac{\sigma \tau_{s}}{\left(1+s \sigma \tau_{s}\right)}
\end{aligned}
$$

resumindo,

$$
G_{S}(s)=\frac{\sigma \tau_{s}}{\left(1+s \sigma \tau_{s}\right)}
$$

Considerando a compensação do pólo dominante, o zero 
do controlador anula o pólo do sistema, logo,

$$
\begin{aligned}
1+s \sigma \tau_{s} & =1+s T_{n} \\
T_{n} & =\sigma \tau_{s}
\end{aligned}
$$

A fonte de tensão estática empregada na alimentação da máquina (inversor PWM-VSI) é implementada utilizandose técnica de modulação escalar regular simétrica (Jacobina, [19]). Esta fonte, pode ser aproximada por um elemento $Z O H$ (figura 3), cuja função de transferência é,

$$
G_{F}(s)=\frac{K_{f}}{\left(1+s T_{p}\right)}
$$

neste caso, $K_{f}(=1)$ é o ganho da fonte, considerado unitário. Definindo a freqüência de canto $\omega_{c a}\left(=\frac{\sigma \tau_{s}}{T_{i}}\right)$ e fazendo a freqüência de corte $\omega_{c o}=\omega_{c a} / 2=\frac{1}{2 T_{p}} \operatorname{com} T p=\frac{t_{a}}{2}$.

A função de transferência do sistema completo (controlador + sistema + fonte) em malha aberta resulta,

$$
G_{o}(s)=\frac{\sigma \tau_{s}}{s T_{i}\left(1+s T_{p}\right)}
$$

A função de transferência do sistema completo em malha fechada é,

$$
G_{c}(s)=\frac{k_{1}}{s^{2}+s k_{2}+k_{1}}
$$

onde $k_{1}=\frac{\sigma \tau_{s}}{T_{i} T_{p}}$ e $k_{2}=\frac{1}{T_{p}}$.

Os ganhos proporcional e integral são calculados, respectivamente, na forma [18],

$$
\begin{aligned}
T_{i} & =4 \sigma \tau_{s} T_{p} \\
T_{n} & =\sigma \tau_{s} \\
K_{i} & =\frac{t_{a}}{T_{i}} \\
K_{p} & =\left(\frac{T_{n}-T_{p}}{T_{i}}\right)
\end{aligned}
$$

\section{RESULTADOS DE SIMULAÇÕES E EXPERIMENTAIS}

Alguns resultados de simulações e experimentais obtidos são apresentados na seqüência. A avaliação do desempenho dinâmico do sistema de acionamento, foi realizada por meio de um ensaio dinâmico de caracterização, considerando as condições de operação apresentadas na tabela (1). As demais condições consideradas para simulação e ensaios da estratégia de controle de velocidade foram: velocidade inicial da máquina $\omega_{r}=0$, tempo de realização do ensaio $t=12,8 \mathrm{~s}$ e temperatura normal de funcionamento. No ensaio em baixa velocidade, a máquina é excitada, a partir de fluxos iniciais nulos, segundo uma rampa de referência de 0,02 s de duração.

Os resultados experimentais foram obtidos na plataforma de testes composta de um microcomputador compatível $\mathrm{IBM}^{\circledR} / \mathrm{PC}$, com processador Pentium ${ }^{\circledR} 233 \mathrm{MHz}$; módulo máquina (composto de máquina $\mathrm{CA}$ assíncrona trifásica, máquina CC funcionando como carga e um sensor ótico de posição de 9 bits acoplado ao eixo da máquina para medição da velocidade); conversor de freqüência retificador + inversor Semikron ${ }^{\circledR}$ (retificador não controlado a diodos com barramento CC de $700 \mathrm{~V}$ e inversor trifásico a $I G B T$ podendo operar com freqüência de chaveamento de até $20 \mathrm{KHz}$; placa multifunção que realiza as funções de aquisição e comando do inversor opera com clock de $10 \mathrm{MHz}$. Os conversores A/D utilizados são de 10 bits com tempo de conversão de $25 \mu \mathrm{s}$.

\begin{tabular}{|l|l|l|}
\hline \multicolumn{3}{|c|}{ Perfil de velocidade $\omega_{r}^{*}(\mathrm{rad} . \mathrm{mec} / \mathrm{s})$} \\
\hline$t=0,00 \mathrm{~s}$ & $\Longrightarrow$ & $\omega_{r}^{*}=150,0$ \\
\hline$t=3,20 \mathrm{~s}$ & $\Longrightarrow$ & $\omega_{r}^{*}=-100,0$ \\
\hline$t=5,60 \mathrm{~s}$ & $\Longrightarrow$ & $\omega_{r}^{*}=-50,0$ \\
\hline$t=8,00 \mathrm{~s}$ & $\Longrightarrow$ & $\omega_{r}^{*}=0,0$ \\
\hline$t=8,80 \mathrm{~s}$ & $\Longrightarrow$ & $\omega_{r}^{*}=\frac{150,0 *(t-8,80)}{4,00}$ \\
\hline \multicolumn{2}{|c|}{ Período de amostragem $t_{a}(\mu \mathrm{s})$} \\
\hline \multicolumn{2}{|c|}{$t_{a}=100,0$} \\
\hline Freqüência chaveamento inversor $(\mathrm{KHz})$ \\
\hline \multicolumn{2}{|c|}{$f_{c h a v}=10,0$} \\
\hline Tensão no barramento CC - $E_{c c}(\mathrm{~V})$ \\
\hline \multicolumn{2}{c|}{$E_{c c}=300,0$} \\
\hline Fluxo de referência $\phi_{s}^{*}(\mathrm{~Wb})$ \\
\hline \multicolumn{2}{|c|}{$\phi_{s}^{*}=0,4$} \\
\hline
\end{tabular}

Tabela 1 Condições gerais para ensaio da estratégia de controle de velocidade sem sensor mecânico.

A figura 4 apresenta os resultados obtidos de simulações. Na curva (a) são traçados a velocidade de referência $\omega_{r}^{*}$ (linha tracejada) e a velocidade estimada $\widehat{\omega}_{r}$ (linha cheia); em (b) os fluxos estatórico de referência $\phi_{s}^{*}$ e o estimado $\widehat{\phi}_{s}$; e finalizando, em (c) o erro existente entre a velocidade de referência e a estimada $\Delta \omega_{r}\left(=\omega_{r}^{*}-\widehat{\omega}_{r}\right)$. Pode-se observar que a velocidade estimada segue a referência com rapidez satisfatória, sem apresentar overshoot e sem oscilações em regime permanente. Mesmo na pior condição de teste, quando é aplicado no instante $t=8,80 \mathrm{~s}$ uma rampa como referência, a estratégia corresponde às expectativas. O fluxo estimado segue sua referência mesmo frente a variações da referência de velocidade.

Os resultados experimentais são apresentados nas figuras 5, 6 e 7 . Primeiramente na figura 5, curva (a), são traçadas as curvas da velocidade de referência $\omega_{r}^{*}$ (linha tracejada) e a estimada $\widehat{\omega}_{r}$ (linha cheia); na curva (b), o erro $\Delta \omega\left(=\omega_{r}^{*}-\widehat{\omega}_{r}\right)$ existente entre elas; e na curva (c) os fluxos de referência $\phi_{s}^{*}$ e estimado $\widehat{\phi}_{s}$.

Pode-se verificar a exeqüibilidade do método considerando que a velocidade estimada segue perfeita e rapidamente sua referência, tanto nos degraus quanto na rampa, sem overshoots ou ripple em regime permanente. O fluxo estatórico também apresenta bom comportamento sem oscilações quando de variações da velocidade de referência, mantendo o valor desejado. Os valores obtidos através de ensaios comprovam os resultados obtidos através de simulações.

Na figura 6, curva (a) observa-se a velocidade de referência $\omega_{r}^{*}$ e a velocidade $\omega_{r}$ medida. Na curva (b) é apresentado o erro $\Delta \omega\left(=\omega_{r}^{*}-\omega_{r}\right)$ existente entre elas e em (c) os fluxos de referência $\phi_{s}^{*}$ e estimado $\widehat{\phi}_{s}$. Verifica-se que a velocidade medida tem comportamento similar à velocidade estimada. Para confirmar esta conclusão, na figura 7(a) são 

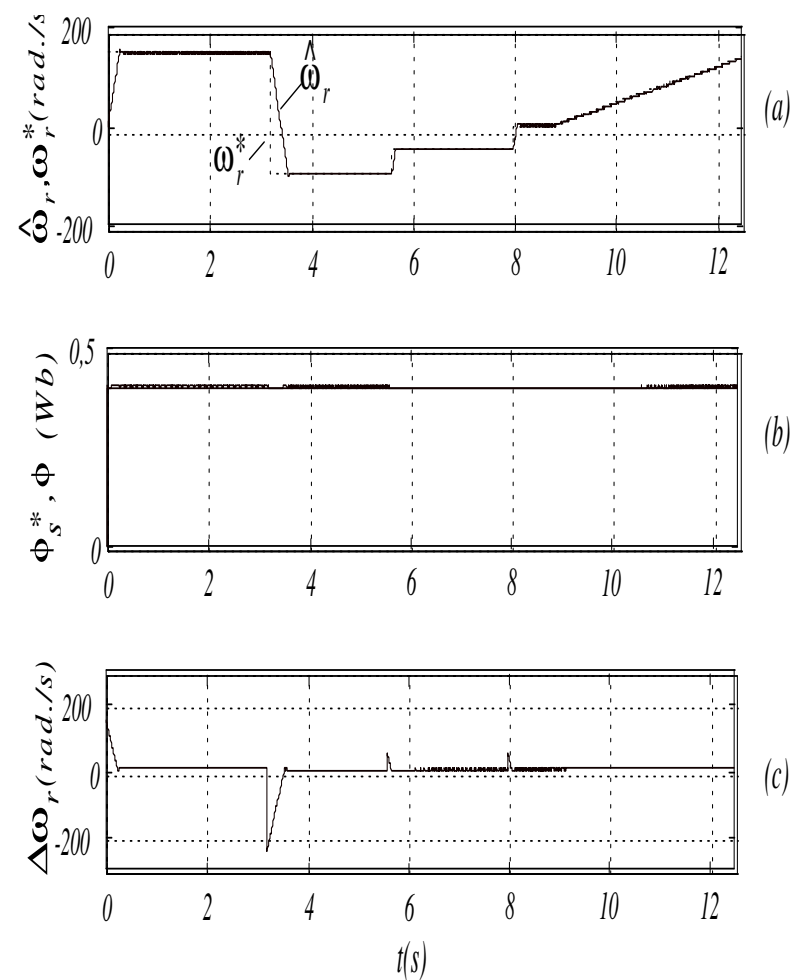

Figura 4 (a) Velocidade rotórica de referência/estimada; (b) erro de velocidade; e, (c) fluxo de refência/estimado.
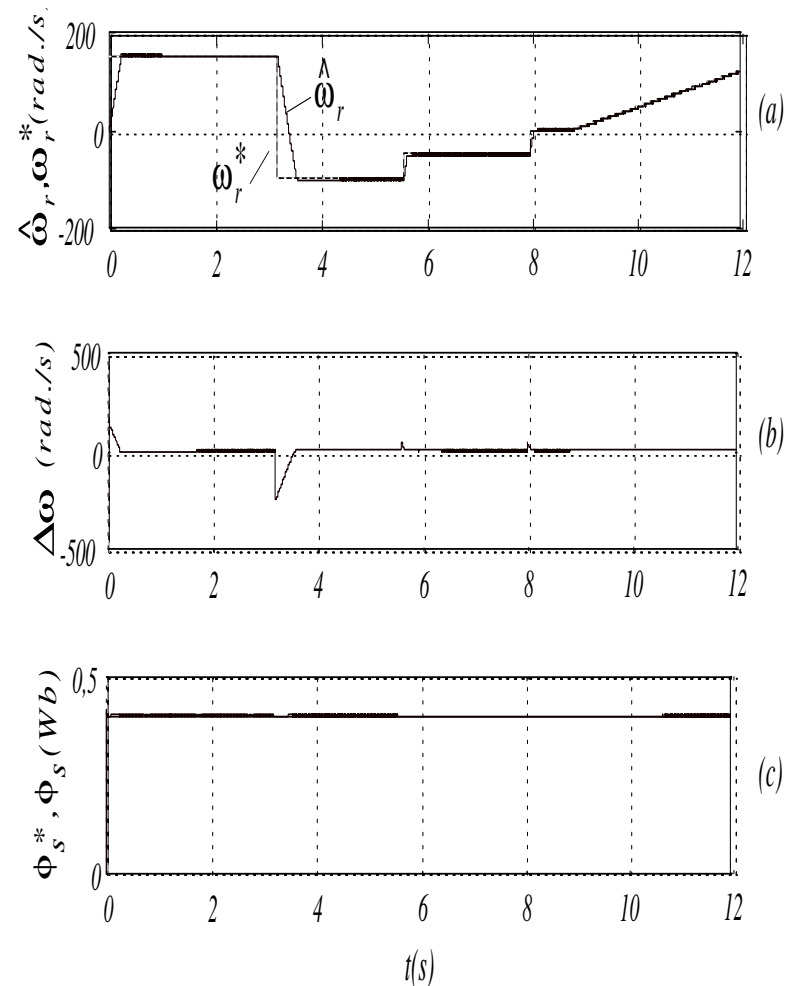

Figura 5 (a) Velocidade rotórica de referência e estimada; (b) erro de velocidade; e, (c) fluxo de refência e estimado.

sobrepostas as curvas da velocidade estimada $\widehat{\omega}_{r}$ (linha tracejada) e a medida $\omega_{r}$ (linha cheia). Em (b) é traçado o erro $\Delta \omega\left(=\widehat{\omega}_{r}^{*}-\omega_{r}\right)$ existente entre elas. Os resultados obtidos demonstraram ser compatíveis.

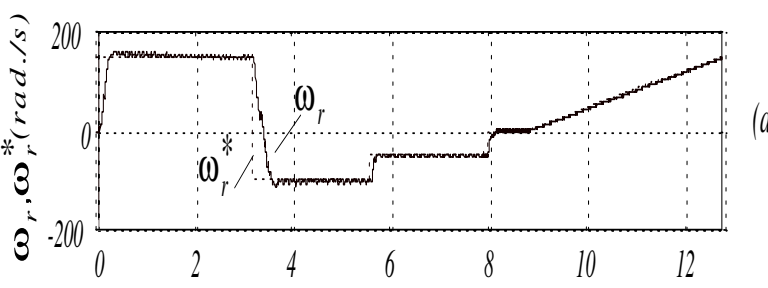

(a)
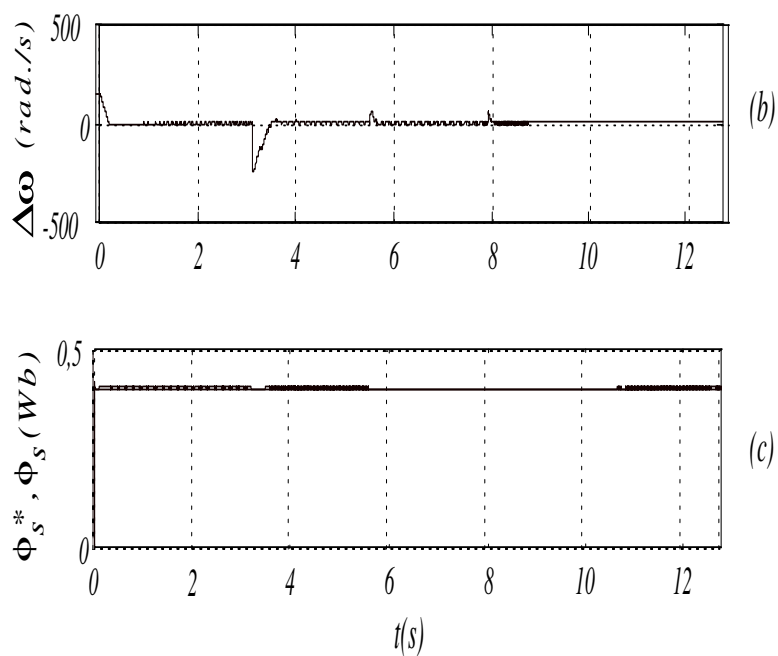

Figura 6 (a) Velocidade rotórica de referência/medida; (b) erro de velocidade; e, (c) fluxo de refência/estimado.

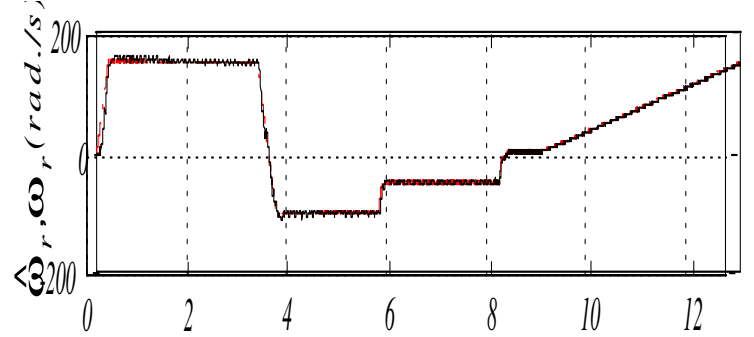

(a)

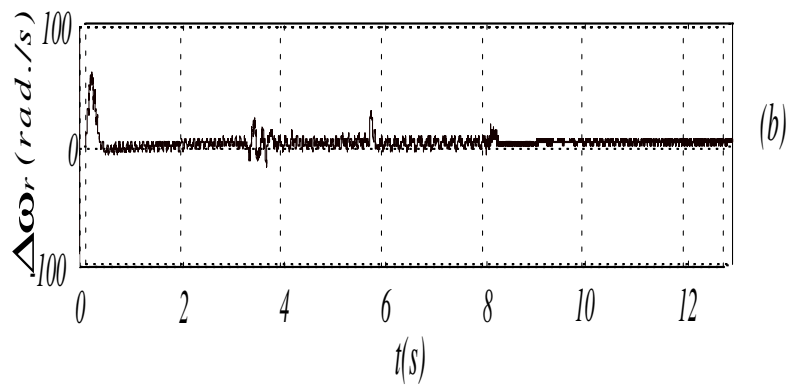

Figura 7 (a) Velocidade rotórica medida/estimada; e, (b) erro de velocidade.

\section{CONCLUSÕES}

Neste artigo é apresentada uma estratégia de controle de velocidade utilizando estratégia $D T C$ para máquinas assíncronas. Os resultados obtidos com a estratégia, demonstraram ser esta, uma alternativa atraente para a implementação de sistemas de acionamentos com máquinas assíncronas de baixo custo e desempenho comparável as estratégias de controle com orientação direta pelo campo $(D F O C)$. 
A estratégia apresentou:

1. Boas respostas no controle da velocidade, tanto em regime transitório (rápido seguimento das referências) como em regime permanente (oscilações de pequena amplitude), sem variações na magnetização da máquina (controle do fluxo estatórico);

2. Simplicidade de implementação, caracterizada por ser totalmente discreta, utilizando controladores PI convencionais, calculados utilizando critério de amortecimento ótimo e compensação do pólo dominante. Além disso, a estratégia é implementada utilizando apenas dois sensores para medição da corrente estatórica;

A freqüência de escorregamento $\widehat{\omega}_{a r}$ é obtida através da estimação, do vetor fluxo estatórico e do conjugado eletromagnético, e utilizando o modelo $d, q$ da máquina assíncrona em regime permanente. A freqüência angular síncrona $\omega_{a}^{*}$ é estimada utilizando um controlador PI. Definindo o conjugado eletromagnético desejado, o controlador PI determinará a freqüência angular síncrona necessária para que este conjugado seja obtido, acelerando ou desacelerando o vetor fluxo estatórico. Por sua vez, a velocidade rotórica estimada é obtida subtraindo $\widehat{\omega}_{a r}$ de $\omega_{a}^{*}$.

Observando-se o diagrama esquemático da estratégia (figura 2), verifica-se que as tensões estatóricas utilizadas no observador de estados são as tensões de referência na saída dos controladores de fluxo. Esta implementação é interessante pois elimina a necessidade de mais dois sensores para a medição das tensões o que reduz o custo financeiro do sistema. Contudo, isto só é possível quando a fonte de tensão é de boa qualidade e não ocorram oscilações no barramento CC.

Os principais problemas enfrentados para obtenção dos resultados experimentais foram com relação a medição das correntes estatóricas (ruídos) e da estimação do fluxo estatórico. Para solucionar o problema de medição das correntes, estas foram filtradas utilizando um filtro antialiasing analógico de $2^{a}$ ordem. O fluxo estatórico foi estimado utilizando um observador em malha aberta modelo em tensão e os fluxo rotóricos obtidos por relação. Este observador apresentou, como era de se esperar problemas em baixas velocidades e de integração para tempos longos de funcionamento.

Contudo, os resultados obtidos com a estratégia através de simulações e experimentalmente demonstraram ser esta estratégia um alternativa viável para a implementação de sistemas de acionamento com máquinas assíncronas de baixo custo e bom desempenho para o controle de velocidade.

Os parâmetros da máquina, foram obtidos através de catálogo do fabricante (EBERLE $\left.{ }^{\circledR}\right)$ e através de ensaios clássicos e métodos de estimação de parâmetros.

\section{REFERÊNCIAS BIBLIOGRÁFICAS}

[1] Leonhard, W. Control of eletrical drives. Springer Verlag, New York - USA, 1996.

[2] Falb, P. e Wollovich, W. Decoupling in the design and synthesis of multivariable control systems. IEEE Trans. on Automatic Control, AC-12, 651-659, december 1967.
APÊNDICE A - PARÂMETROS DA MÁQUINA

\begin{tabular}{|l|l|}
\hline Potência & $P_{a}=0,2453 \mathrm{~kW}$ \\
\hline Corrente nominal & $I_{s}=0.8 \mathrm{~A} / \mathrm{Y}-380 \mathrm{~V}$ \\
\hline Velocidade nominal & $w_{m}=1670 \mathrm{rpm}$ \\
\hline Freqüência nominal & $f=60 \mathrm{~Hz}$ \\
\hline Conjugado nominal & $C_{n}=0,15 \mathrm{Nm}$ \\
\hline Constante de inércia & $J_{m}=0,0137 \mathrm{Nm}^{2}$ \\
\hline Coeficiente de atrito & $F_{a}=0,0075 \mathrm{Nm}$ \\
\hline Resistência estatórica & $r_{s}=26,77 \Omega$ \\
\hline Resistência rotórica & $r_{r}=26,37 \Omega$ \\
\hline Indutância estatórica & $l_{s}=0,5211 \mathrm{H}$ \\
\hline Indutância rotórica & $l_{r}=0,5256 \mathrm{H}$ \\
\hline Indutância mútua & $l_{m}=0,4977 \mathrm{H}$ \\
\hline Número de pares de pólos & $P=2$ \\
\hline
\end{tabular}

Tabela 2 Parâmetros da máquina assíncrona.

[3] Bose, B. K. Microcomputer Control of Power Electronics and Drives. IEEE - Press, New York - USA, 1987.

[4] Lima, A. M. N. Comande numérique d 'une machine asynchrone: Controle du flux par filtrage stochastique Variation de la vitesse et de la position. Thése de docteur de l'inp, INP - Toulouse - France, INP - Toulouse - France, juillet 1989.

[5] Hasse, K. On the dynamics of speed control of static ac drives with squirrel-cage induction machines. Phd thesis, TH Darmstadt, Darmstadt - Germain, 1969.

[6] Blaschke, F. The method of field orientation for control of three phase machines. Phd thesis, TU Braunschweig, Braunschweig - Germain, 1974.

[7] Takahashi, I. e Noguchi, T. A new quick response and high efficiency control strategy of an induction motor. IEEE Trans. on Industry Applications, 22, n. 5, 820827, sep/oct 1986.

[8] Depenbrock, M. Direct self-control (dsc) of inverter-fed induction machines. IEEE Trans. on Power Electronics, 3, n. 4, 420-429, october 1988.

[9] Boldea, I. e Nasar, S. A. Vector Control of AC Drives. CRC Press, Boca Raton - Florida - USA, first edn., 1992.

[10] Habetler, T. e Divan, D. Control strategies for direct torque control using discrete pulse modulation. IEEE Trans. on Industry Applications, 27, n. 5, 893-901, sep/oct 1991.

[11] Mir, S., Elbuluk, M. e Zinger, D. Fuzzy implementation of direct self control of induction machines. IEEE Trans. on Industry Applications, 30, n. 3, 729-735, may/june 1994.

[12] Salvadori, F., Jacobina, C., Lima, A. e Oliveira, T. Acionamento da máquina assíncrona utilizando estratégia de controle direto de fluxo e conjugado. In: Anais $C O$ BEP'99, Foz do Iguaçu - PR - BR, set 1999. SOBRAEP Sociedade Brasileira de Eletrônica de Potência.

[13] Alger, P. L. Induction Machines - Their Behavior and Uses. Gordon and Breach Publishers, Switzerland, 1995.

[14] Jacobina, C. e Lima, A. Estratégias de controle para sistemas de acionamento com máquinas ass $S B A$ - Controle $\&$ Automação, 7, n. 1, 15-28, jan/abr 1996.

[15] Jansen, P. L. The Integration of State Estimation, Con- 
trol, and Design for Induction Machines. Ph.d. thesis, University of Wisconsin - Madison, Wisconsin - Madison - USA, june 1993.

[16] Lorenz, R. D., Lipo, T. A. e Novotny, D. W. Motion control with induction motors. Proceedigns of IEEE: especial issue on power electronic and motion control, 82, n. 8, 1215-1240, august 1994.

[17] de Souza Ribeiro, L., Jacobina, C. e Lima, A. Estimação da velocidade da máquina assíncrona em acionamentos estáticos - parte i: Revisão e classificação. In: Anais COBEP'97, p. 486-497, Belo Horizonte, novembro 1997.

[18] Bühler, H. Eletronique de réglage et comande. Ed. Dunod, Lausane - France, 1979.

[19] Jacobina, C. B. Contribuição a estimação e ao controle em acionamentos estáticos com máquinas assíncrona. Tese concurso professor titular, UFPB/CCT/DEE, Campus II - Campina Grande - PB, outubro 1995.

\section{DADOS BIOGRÁFICOS}

Fabiano Salvadori, nascido em 1962 em Tapera, Rio Grande do Sul, é engenheiro eletricista (1988) pela Universidade Católica de Pelotas (UCPel), mestre (1991) e doutor (2000) em Engenharia Elétrica pela Universidade Federal da Paraíba. Desde fevereiro de 1992 é professor do Departamento de Tecnologia, curso de Engenharia Elétrica, da Universidade Regional do Noroeste do Estado do Rio Grande do Sul (UNIJUÍ). Suas áreas de interesse incluem Eletrônica de Potência e Acionamento de Máquinas Elétricas.

Gideon Villar Leandro, nascido em 1965 em Américo de Campos, São Paulo, é engenheiro eletricista (1989) pela Universidade Estadual Paulista (UNESP), mestre (1992) em Engenharia Elétrica pela Universidade Federal da Paraíba (UFPB) e doutor (2000) em Engenharia Elétrica pela Universidade Estadual de Campinas (UNICAMP). Desde 1993 é professor do Departamento de Tecnologia da Universidade Regional do Noroeste do Estado do Rio Grande do Sul (UNIJUÍ). Suas áreas de interesse são Sistemas de Controle, Identificação de Sistemas e Sistemas a Eventos Discretos.

Cursino B. Jacobina e Antônio Marcus N. Lima, tem suas biografias apresentdas no quarto artigo desta revista. 